\title{
Diseños de Investigación
}

\section{Research Designs}

\author{
Ana J. Monjarás-Ávila ${ }^{a}$, Ana K. Bazán-Suárez ${ }^{b}, Z^{2}$ aida K. Pacheco-Martínez ${ }^{c}$, \\ José A.Rivera-Gonzaga ${ }^{d}$, Juan E. Zamarripa-Calderón ${ }^{e}$, Carlos E. Cuevas-Suárez ${ }^{f}$.
}

\begin{abstract}
:
Research is a reflexive, systematic, controlled and critical procedure that aims to discover or interpret the facts of a phenomenon. The research design constitutes the general plan of the researcher to obtain answers to his questions or to verify the research hypothesis, in addition, it stipulates the fundamental and specific structure of the intervention.
\end{abstract}

Keywords:

Research, methodology, design, levels.

\section{Resumen:}

La investigación es un procedimiento reflexivo, sistemático, controlado y crítico que tiene por finalidad descubrir o interpretar los hechos de un fenómeno. El diseño de investigación constituye el plan general del investigador para obtener respuestas a sus interrogantes o comprobar la hipótesis de investigación, además, estipula la estructura fundamental y especifica de la intervención.

Palabras Clave:

Investigación, metodología, diseño, niveles

\section{Introducción}

Los diseños de investigación son estrategias metodológicas para alcanzar propósitos investigativos, esto se refiere a la expresión de una planificación que comparte características en común.

A nivel de investigación, puede haber ideas parecidas, sin embargo, las estrategias para llegar al objetivo final son distintas, ya que existen limitaciones a nivel instrumento y debido a ciertas características se cambia todo el panorama.

Ej. Un par de estudios observacionales, descriptivos, transversales, prospectivos, pero con diferente marco muestral.

El diseño de investigación se describe por sus características en niveles de investigación y tipos de estudio.
Niveles de la investigación:

Se refiere a la profundidad con que se abordará un objeto de estudio, se caracterizan por un principio y se desarrollan en un protocolo según la necesidad de conocer el planteamiento del problema de investigación.

Estos se describen a continuación.

1.- Exploratorio: Se utiliza cuando un problema aún no ha sido abordado o suficientemente estudiada, su función es el reconocimiento e identificación de problemas, se trata de investigación cualitativa.

Ej. Identificación de la enfermedad periodontal.

2.- Descriptivo: Se efectúa cuando se describen los componentes principales del objeto de estudio. Se

\footnotetext{
a Autor de Correspondencia, Universidad Autónoma del Estado de Hidalgo, Instituto de Ciencias de la Salud, ORCID: 0000-0002-5916-6510, Email: ana_monjaraz@uaeh.edu.mx

${ }^{\text {b } U n i v e r s i d a d ~ A u t o ́ n o m a ~ d e l ~ E s t a d o ~ d e ~ H i d a l g o, ~ I n s t i t u t o ~ d e ~ C i e n c i a s ~ d e ~ l a ~ S a l u d, ~ E m a i l: ~ k a r e n b a z a n n @ ~ g m a i l . c o m ~}$

${ }^{c}$ Universidad Autónoma del Estado de Hidalgo, Instituto de Ciencias de la Salud, Email: karen_tuzos@hotmail.com

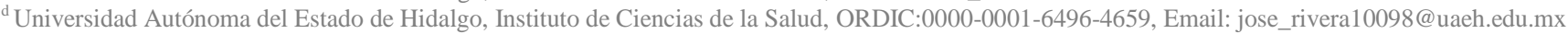

${ }^{\mathrm{e}}$ Universidad Autónoma del Estado de Hidalgo, Instituto de Ciencias de la Salud, ORDIC:0000-0001-5830-5550, Email: eliezerz@uaeh.edu.mx

${ }^{\mathrm{f}}$ Universidad Autónoma del Estado de Hidalgo, Instituto de Ciencias de la Salud,ORCID:0000-0002-2759-8984, Email: cecuevas@uaeh.edu.mx
} 
describen frecuencias y promedios, y se estiman parámetros con intervalos de confianza.

Ej. Prevalencia de la enfermedad periodontal.

3.- Relacional: Es aquel que persigue medir el grado de relación existente entre dos o más variables. La estadística es bivariado, no permite hacer asociaciones (Chi cuadrado), ni correlaciones (Correlación de Pearson).

Ej. Factores de riesgo de la enfermedad periodontal.

4.- Explicativo: No sólo describe un problema, sino que intenta encontrar las causas del mismo, explicando el comportamiento de una variable en función de otra. Cumplen con criterios de causalidad, el control estadístico es multivariado para descartar asociaciones aleatorias entre variables independientes y dependientes.

Ej. Causas de la enfermedad periodontal.

5.- Predictivo: Se encarga de la estimación de eventos adversos como la enfermedad o en función al tiempo, se aplican técnicas estadísticas específicas.

Ej. Pronóstico de la enfermedad periodontal.

6.- Aplicativo: Plantea resolver problemas o intervenir en la historia natural de la enfermedad. Las técnicas estadísticas evalúan el éxito de la intervención en cuanto a proceso, resultados e impacto.

Ej. Tratamiento de la enfermedad periodontal.

\section{- Tipos de estudios:}

Determina la manera de cómo el investigador abordará el objeto de estudio, de acuerdo con las técnicas, métodos, instrumentos y procedimientos propios de cada uno.

- En base a las características de sus datos:

a) Cualitativo: obtención de datos no cuantificables sustraídos de la observación, aunque ofrece mucha información sus datos son subjetivos y no permiten una explicación clara del fenómeno, ya que se centra en aspectos descriptivos.

b) Cuantitativo: análisis a través de diferentes procedimientos sustraídos de la medición, permitiendo un mayor control e inferencia siendo posible obtener explicaciones a partir de una hipótesis, sus resultados se basan en la estadística.
- En base a su dimensión temporal:

a) Transversales: comparación de determinadas características o situaciones en diferentes sujetos en un momento concreto, compartiendo la misma temporalidad.

b) Longitudinales: se caracteriza por realizar un seguimiento a los mismos sujetos de estudio por un largo periodo de tiempo, esto permite ver la evolución de características y variables observadas.

- En base a su intervención:

a) Observacionales: Aquellos que no tiene intervención por parte del investigador.

Ej. Prevalencia de microorganismos de absceso periodontal en pacientes diabéticos

A su vez se subdividen en:

a. Descriptivos: reporte de casos, serie de casos, estudios transversales.

b. Analíticos: estudio de casos y controles y el estudio de cohorte.

b) Experimentales: Aquellos en los que interviene el investigador sobre una variable para modificar un resultado, con fines de observar una relación causal. Ej. Comparación de microorganismos de enfermedad periodontal antes y después al uso de enjuague bucal con clorhexidina al $2 \%$.

- En base a la obtención de sus datos:

a) Prospectivos: El inicio del estudio es anterior a los hechos estudiados, los datos se recopilan a medida que se van obteniendo.

b) Retrospectivos: El inicio del estudio es posterior a los hechos estudiados, los datos se recopilan de archivos o entrevistas sobre hechos sucedidos. 


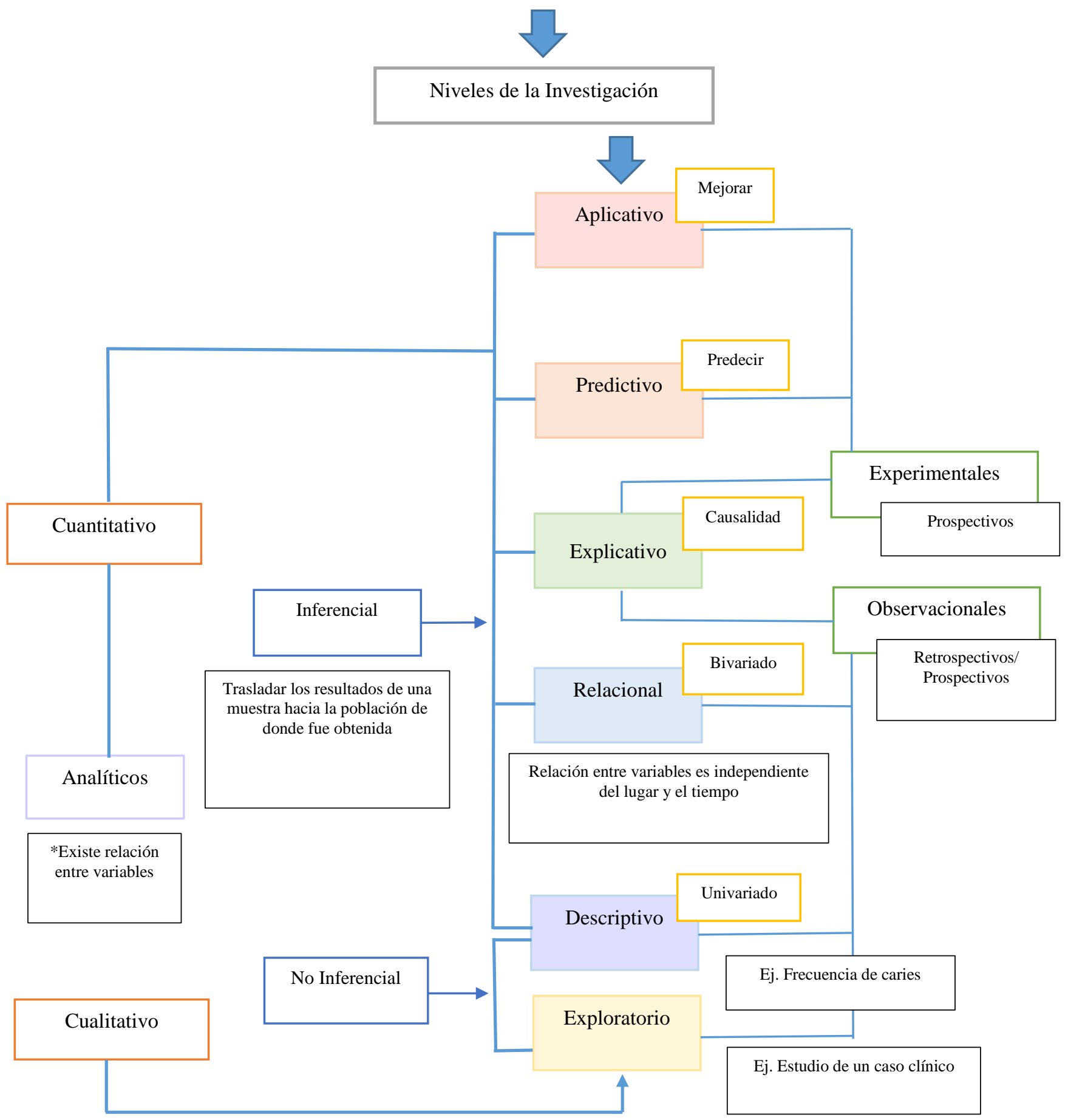




\section{Conclusión:}

Un buen diseño de investigación deber ser apropiado para la pregunta que se ha planteado el investigador. Debe también, llevar al mínimo o evitar los sesgos que puedan distorsionar los resultados del estudio. Adicionalmente, un buen diseño trata de mejorar la precisión de la investigación, lo cual denota la sensibilidad para detectar los efectos de la variable independiente, en relación con los efectos de las variables extrañas. Finalmente, el diseño debe contemplar de manera apropiada el aspecto del poder de la investigación, es decir, la capacidad del diseño para crear el máximo contraste entre los grupos de comparación.

\section{Referencias}

[1] Castillero, O. (s.f.). Psicología y Mente. Obtenido de: https://psicologiaymente.net/miscelanea/tipos-de-investigacion

[2] Siqueira, C. (2017). Universia Costa Rica. Obtenido de http://noticias.universia.cr/educacion/noticia/2017/09/04/1155475/tiposinvestigacion-descriptiva-exploratoria-explicativa.html

[3] Supo, J. (2013). SlideShare. Obtenido de https://es.slideshare.net/josesupo/niveles-de-investigacion-15895478 